\title{
Contribuição de folhas na formação da serrapilheira e no retorno de nutrientes em floresta de transição no norte de Mato Grosso
}

\author{
Carlos José da SILVA, Francisco de Almeida LOBO$^{2}$, Monica Elisa BLEICH ${ }^{3}$, Luciana SANCHES ${ }^{4}$ \\ RESUMO
}

Este estudo foi desenvolvido numa área de floresta de transição Amazônia-Cerrado no norte de Mato Grosso. Teve como objetivo quantificar a dinâmica da produção e acúmulo e as partes constituintes da serrapilheira, bem como a participação das folhas no retorno do nitrogênio, fósforo e carbono ao solo por meio da decomposição. Foram coletados mensalmente durante um ano dados de serrapilheira total produzida, serrapilheira total acumulada no solo e avaliada a decomposição de folhas no período de seca e de chuvas. A produção de serrapilheira apresentou variaçóes sazonais com maiores valores no período seco e predomínio da fração foliar durante todo o ano. Por outro lado, a serrapilheira acumulada não apresentou diferenças significativas entre o período de seca e o chuvoso como também correlação significativa com a serrapilheira produzida. A decomposição da serrapilheira exclusiva de folhas foi mais intensa no período chuvoso e em ordem decrescente de espécies, em T. schomburgkii, Thyrsodium sp, B. lactescens e P. sagotianum. A espécie $P$. sagotianum tanto no período seco quanto no chuvoso apresentou maior resistência à decomposição. O potencial de retorno de nitrogênio ao solo foi da ordem de $70,3 \mathrm{~kg} \cdot \mathrm{ha}^{-1} \cdot \mathrm{ano}^{-1}$, para o fósforo, de $76,1 \mathrm{~kg} \cdot \mathrm{ha}^{-1} \cdot$ ano $^{-1} \mathrm{e}$ para o carbono, de 3,5 ton.ha ${ }^{-1} \cdot$ ano $^{-1}$. Houve uma correlação entre a decomposição exclusiva de folhas e o retorno de nitrogênio e fósforo para todas as espécies estudadas no período de seca. No período chuvoso foi registrada correlação significativa somente para nitrogênio e o carbono para a espécie $P$. sagotianum.

PALAVRAS-CHAVE: ciclagem de nutrientes, serrapilheira, floresta de transição.

\section{Leaf contribution to litterfall and litterpool and nutrients recycling in transition forest in Mato Grosso}

\section{ABSTRACT}

This study was conducted in an area of Amazonia-Cerado transition forest in the noirth of Mato Grosso state, Brazil. The main objectives of the study was to quantify the variability of litter fall and litter pool biomass (leaves, flowers, fruits, and wood), and the participation of the leaves in the return of nitrogen, phosphorus and carbon to the soil through decomposition. Litter was collected monthly during one year and leaves decomposition was analyzed during the dry and rainy seasons. Litter fall varied along the year with higher values in the dry season and predominance of the leaf fraction. Nevertheless, there was no significant difference between dry and rainy seasons for litter pool as well as significant correlation between litter pool and litter fall. Decomposition of leaves was more intense in the rainy season in order of decreasing species: T. schomburgkii, Thyrsodium sp, B. lactescens and $P$. sagotianum. The species $P$. sagotianum in both dry and rainy seasons showed most slowly decomposition. The potential return was $70.3 \mathrm{~kg} \cdot \mathrm{ha}^{-1}$.year ${ }^{-1}$ for nitrogen, $76.1 \mathrm{~kg} \cdot \mathrm{ha}^{-1}$.years ${ }^{-1}$ for phosphorus, 3.5 ton.ha ${ }^{-1}$. years $^{-1}$ for carbon. There was significant correlation between leave decomposition and nitrogen and phosphorus return for all species analyzed in the dry season. However, it was found significant correlation only for the carbon and the nitrogen for the species $P$. sagotianum in the rainy season.

KEY WORDS: Nutrients cycling, Litter fall and litter pool, transition forest

\footnotetext{
1 Universidade do Estado de Mato Grosso, Campus de Alta Floresta, Departamento de Engenharia Florestal. E-mail: cjsnx@yahoo.com.br

2 Universidade Federal de Mato Grosso, Campus de Cuiabá. E-mail: f_a_lobo@pq.cnpq.br

3 Universidade do Estado de Mato Grosso, Campus de Alta Floresta, Departamento de Engenharia Florestal. E-mail: monicaeb@unemat.br

${ }^{4}$ Universidade Federal de Mato Grosso, Departamento de Engenharia Sanitária e Ambiental.
} 


\section{INTRODUÇÃO}

Muitos estudos têm descrito como a diversidade de plantas influencia as propriedades do ecossistema. Alguns destes estudos mostram que a produção de biomassa vegetal e a disponibilidade de nutrientes no solo estão positivamente relacionadas com a diversidade de espécies de plantas no ecossistema (Naeem et al., 1995; Tilman et al., 1996). Partindo deste princípio, a composição da serrapilheira tem apresentado grande importância no estudo das propriedades dos ecossistemas.

Os ecossistemas de florestas tropicais em geral apresentam produção contínua de serrapilheira no decorrer do ano, sendo que a quantidade produzida nas diferentes épocas depende do tipo de vegetação considerada (Leitão-Filho et al., 1993; Rodrigues e Leitão Filho, 2001). Segundo Martins e Rodrigues (1999) e Vital et al. (2004), o estudo da ciclagem de nutrientes minerais, via serrapilheira, é fundamental para o conhecimento da estrutura e funcionamento de ecossistemas florestais.

Os solos sob a floresta de transição Amazônia-Cerrado apresentam uma fertilidade natural muito baixa, de maneira que as espécies vegetais necessitam contar, para o seu pleno desenvolvimento, com a ciclagem de nutrientes que envolve a decomposição da matéria orgânica. Entretanto, a produção de serrapilheira e sua decomposição são fenômenos que apenas recentemente vêm sendo estudados nesse ecossistema.

Estudos que buscam compreender o funcionamento destes sistemas, com o mesmo comportamento climático, são de grande importância para a compreensão e determinação de modelos regionais. Silva et al. (2007) registraram maior produção de serrapilheira na floresta de transição em comparação com fitofisionomias do cerrado no Mato Grosso, e em ambas as áreas a produção foi maior na estação seca. Em outros estados, estudos de produção de serrapilheira realizados por Klinge e Rodrigues (1968); Luizão e Schubart (1987), Boinskis (1989), Dantas e Phillipsson (1989), Luizão (1989), Morellato e Leitão Filho (1995), Rodrigues et al. (2000) e Vital et al. (2004) também observaram máxima produção de serrapilheira no período de seca. Em mata de galeria no sudoeste de Mato Grosso, De Maman (2006) registrou maior produção e acúmulo de serrapilheira do que no cerradão, e maior decomposição da serrapilheira (folhas) no período chuvoso.

Existe a necessidade de realização de mais trabalhos para a avaliação da produção de serrapilheira, da decomposição da matéria orgânica e da ciclagem de nutrientes em diferentes locais da floresta de transição Amazônia-Cerrado, para que se possa ter uma melhor compreensão do efeito das condições particulares de composição florística e do microclima sobre esses fenômenos.
Nesse contexto, o presente trabalho teve como objetivo gerar informações da dinâmica de produção e acúmulo de serrapilheira e as partes constituintes, e quantificar a participação da fração foliar de quatro espécies no retorno de nitrogênio, fósforo e carbono ao solo por meio da decomposição, na floresta de transição Amazônia-Cerrado no norte de Mato Grosso.

\section{MATERIAL E MÉTODOS}

\section{ÁREA DE ESTUDO}

Este estudo foi desenvolvido em uma área remanescente de floresta de transição Amazônia-Cerrado na área de exploração florestal (17.000 ha) pertencente à Fazenda Maracaí. A área está localizada a aproximadamente $50 \mathrm{~km}$ da cidade de Sinop, norte de Mato Grosso, com uma altitude $423 \mathrm{~m}$, onde está instalada uma torre micrometeorológica do Projeto LBA (Grande Experimento Biosfera-Atmosfera na Amazônia) (1 $\left.1^{\circ} 24^{\prime} 43.4^{\prime \prime} S: 55^{\circ} 19^{\prime} 25.7^{\prime \prime} \mathrm{O}\right)$. A temperatura média anual da área é de $26^{\circ} \mathrm{C}$ com pequenas variaçõos nas estações, e precipitação média anual de $2170 \mathrm{~mm}$, variando de zero a 491 $\mathrm{mm}$ ao longo do ano (Figura 1). É uma região de transição entre a floresta tropical úmida e o cerrado, que apresenta uma sazonalidade característica, com uma estação seca de abril a setembro, e uma estação chuvosa de outubro a março.

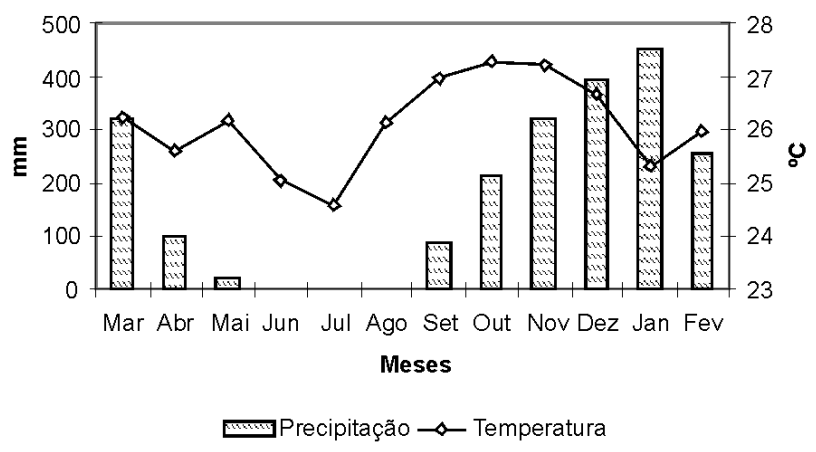

Figura 1-Dados climatológicos obtidos por meio da torre micrometeorológica do LBA na floresta de transição Amazônia-Cerrado durante o período deste estudo.

As espécies de árvores mais comuns em áreas de floresta semidecídua na Bacia Amazônica (Ackerly et al., 1989 apud Sanches et al., 2008), são Protium sagotianum Marchland, Dialium guianense (Aubl.) Sandwith, Hevea brasiliensis Müll. Arg., Brosimum lactescens (S. Moore) C.C. Berg, Cordia alliodora (Ruiz e Pav.) Oken, Tovomita schomburgkii Planch e Triana, e Qualea paraensis Ducke. Existem aproximadamente 94 espécies e 35 famílias de árvores com um diâmetro $\geq 10 \mathrm{~cm}$, no entanto, quase $50 \%$ de todos os indivíduos são das famílias Burseraceae (P. sagotianum), Clusiaceae (T. schomburgkii) e Moraceae (B. lactescens). A altura máxima das copas está entre 25-28 m, a densidade é de 483 ha $^{-1}$ e área basal das árvores é 
de 22,5 $\mathrm{m}^{2} \mathrm{ha}^{-1}$ (Sanches et al., 2008). O índice de área foliar varia entre $4-5 \mathrm{~m}^{2} \mathrm{~m}^{-2}$ na estação chuvosa e $2-3 \mathrm{~m}^{2} \mathrm{~m}^{-2}$ na época da seca refletindo a natureza semidecídua da floresta (Sanches et al., 2005). O solo é do tipo neossolo quartzarênico órtico típico a moderado álico, com profundidade de $50 \mathrm{~cm}$ e textura arenosa com 76,2\% de areia, 6,7 \% de silte e 17,1 $\%$ de argila (Pinto Jr, 2007).

\section{DINÂMICA MENSAL DA PRODUÇÃO DA SERRAPILHEIRA TOTAL}

Para quantificar a produção mensal de serrapilheira total da floresta de maio de 2004 a abril de 2005, foram espalhados 21 coletores quadrados de $1 \mathrm{~m}^{2}$ cada, distantes $20 \mathrm{~m}$ entre si, dentro de um transecto de 20 × 500 m totalizando uma área de 1 ha. Cada coletor tinha fundo de tela de náilon com $2 \mathrm{~mm}$ de abertura de malha, borda de $20 \mathrm{~cm}$ de altura, instalados a 40 $\mathrm{cm}$ acima do solo para evitar possíveis ações decompositoras no material vegetal. $\mathrm{O}$ número de coletores adotado no estudo teve como intuito amostrar de forma representativa 1 ha de floresta de transição.

A serrapilheira dos coletores foi recolhida mensalmente e as amostras foram separadas nas fraçōes folhas, galhos, flores e frutos e em seguida secas em estufa a $70{ }^{\circ} \mathrm{C}$ por 72 horas, determinando-se posteriormente suas respectivas massas. $\mathrm{O}$ valor médio da massa seca de serrapilheira dos 21 coletores foi empregado para avaliar a dinâmica de produção mensal e o total anual produzido.

\section{QUANTIFICAÇÃO DA SERRAPILHEIRA TOTAL ACUMULADA NO SOLO}

$\mathrm{Na}$ mesma área experimental, foram tomadas 21 amostras de serrapilheira total acumulada acima do solo, distantes $20 \mathrm{~m}$ entre si, utilizando-se um quadrante de molde vazado de 0,25 x $0,25 \mathrm{~m}$. O material coletado foi tratado da mesma forma que a serrapilheira produzida.

O valor médio da massa seca de serrapilheira desses 21 quadrantes foi empregado para avaliar a dinâmica de acúmulo mensal e o total anual de serrapilheira remanescente no solo. Para verificar diferenças entre os valores de serrapilheira produzida e serrapilheira remanescente entre os períodos de seca e de chuva, foi utilizado o teste $t$. Para verificar a relação entre os valores de serrapilheira total produzida e de serrapilheira total remanescente quantificados mensalmente, foi realizada uma correlação linear.

\section{MEDIÇÃO DA TAXA DECOMPOSIÇÃO E DO TEMPO MÉDIO DE RETORNO DE NUTRIENTES DE SERRAPILHEIRA EXCLUSIVA DE FOLHAS}

No estudo da taxa de decomposição das folhas, foram selecionadas quatro espécies, por serem aquelas mais dominantes na área de estudo. As espécies foram: Tovomita schomburgkii Planch. e Triana (espécie A, Clusiaceae), Brosimum lactescens (S. Moore) C. C. Berg. (espécie B,
Moraceae), Thyrsodium sp (espécie C, Anacardiaceae) e Protium sagotianum (espécie D, Burseraceae).

O estudo da decomposição e retorno de nutrientes foi efetuado considerando apenas a contribuição relativa da serrapilheira exclusiva das folhas, empregando-se a técnica de bolsas de náilon de $30 \mathrm{~cm}$ x $30 \mathrm{~cm}$ com malha de $2 \mathrm{~mm}$ de diâmetro providas de 3 a $5 \mathrm{~g}$ de folhas e expostas às condições ambientais (Bocock e Gilbert 1957).

Foram realizadas cinco coletas durante o período de seca, aos $15,28,62,92$ e 122 dias e cinco durante o período de chuva, aos 15, 24, 36, 66 e 102 dias após a colocação das bolsas próximo aos pontos de amostragem da serrapilheira produzida e acumulada.

As taxas de decomposição das folhas das quatro espécies selecionadas foram avaliadas pela dinâmica da perda de massa seca, enquanto que as taxas de retorno de nutrientes, pela dinâmica da perda de nitrogênio, fósforo e carbono destas mesmas amostras após a pesagem. As análises de nutrientes seguiram a metodologia descrita por Allen (1989). As folhas utilizadas nas análises foram previamente lavadas para evitar contaminação.

A taxa de decomposição foi estimada por meio da perda de massa seca usando um modelo exponencial de decaimento (Equação 1) e o tempo de decomposição da metade do material original foi determinado pela Equação 2, de acordo com o modelo proposto por Olson (1963).

$$
\begin{aligned}
& \frac{X}{X_{0}}=\exp \left(-K_{f} \cdot t\right) \\
& T_{f_{50 \%}}=\frac{-\ln 0,5}{K_{f}}
\end{aligned}
$$

Onde:

$X=$ matéria seca da serrapilheira exclusiva de folhas no instante $t$ considerado;

$X_{0}=$ matéria seca da serrapilheira exclusiva de folhas inicial;

$t=$ tempo de permanência em campo;

$K_{f}=$ constante de decomposição da serrapilheira exclusiva de folhas;

$T_{f 50 \%}=$ tempo de decomposição de $50 \%$ da serrapilheira exclusiva de folhas.

A análise da variabilidade dos nutrientes foi feita empregando-se dois tipos de regressão. Inicialmente se considerou o mesmo modelo proposto para a análise de decomposição (Equação 1) e, num segundo momento, com base em uma regressão linear do primeiro grau, considerando a fração da concentração atual do nutriente em relação à 
original como variável dependente e o tempo de permanência da amostra em campo, como variável independente. Finalmente, foi realizada uma análise de correlação linear entre as concentraçôes dos nutrientes presentes nas amostras e as respectivas frações de material vegetal remanescente do processo de decomposição das folhas. Esta análise teve por objetivo subsidiar a interpretação do fenômeno da ciclagem de nutrientes originários das folhas bem como da serrapilheira produzida e acumulada.

\section{RESULTADO E DISCUSSÃO}

Dinâmica mensal da produção da serrapilheira produzida em floresta de transição Amazônia-Cerrado

A produção média mensal de serrapilheira total no ano de coleta variou entre 212,9 e $1.334,1 \mathrm{~kg} \cdot \mathrm{ha}^{-1}$ alcançando uma produção anual de $8.992,2 \mathrm{~kg} \cdot \mathrm{ha}^{-1}$, apresentando sazonalidade bem definida, com maior produção no período de seca nos meses de maio a outubro, tendo picos nos meses de agosto e setembro (Figura 2). Houve diferença significativa entre o período seco e chuvoso produção de serrapilheira $(t=3,62$, $P=0,003)$.

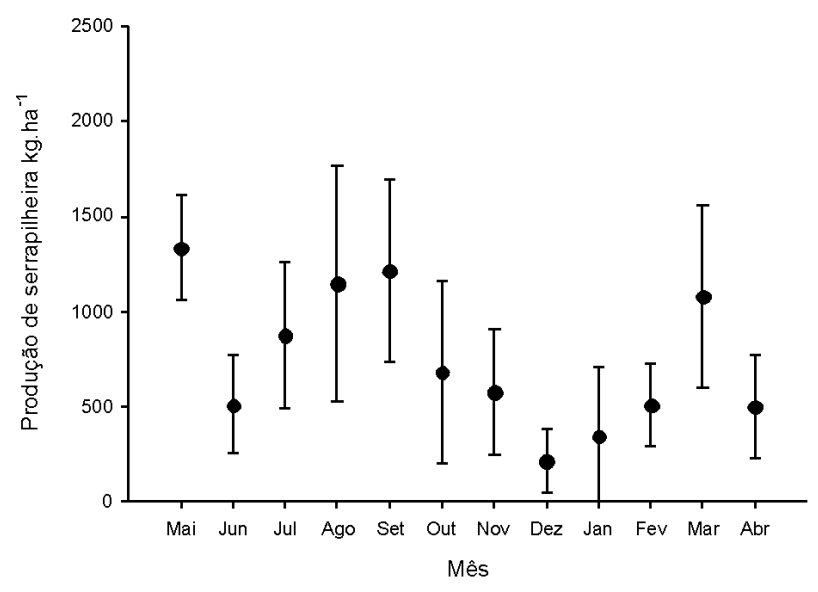

Figura 2 - Produção de serrapilheira na Floresta de Transição AmazôniaCerrado entre maio de 2004 a abril de 2005.

A fração mais representativa na composição da serrapilheira produzida foi composta pelas folhas com $70 \%$, seguida dos galhos $12 \%$, frutos $12 \%$ e flores $6 \%$. Em relação à massa total nota-se que, do ponto de vista temporal, há uma constante queda de folhas, galhos e frutos durante todo o ano, ao passo que durante os meses de fevereiro, março e de abril não se observa queda de flores (Figura 3).

No período chuvoso um pico de produção ocorreu no mês de março $\left(1.080,0 \mathrm{~kg} \cdot \mathrm{ha}^{-1}\right)$, no qual foi registrada a segunda maior contribuição do ano da fração galhos $\left(247,6 \mathrm{~kg} \cdot \mathrm{ha}^{-1}\right)$, que possivelmente é a responsável pelo pico de produção de serrapilheira (Figura 3).

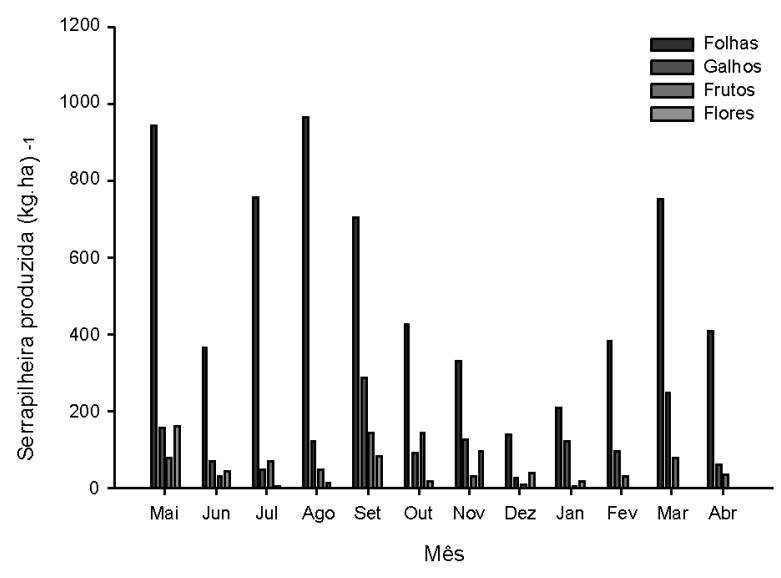

Figura 3 - Frações da serrapilheira produzida na Floresta de Transição Amazônia-Cerrado entre maio de 2004 a abril de 2005.

Resultados semelhantes foram encontrados por Martins e Rodrigues (1999) em floresta estacional semidecidual, por Vital et al. (2004) em floresta ripária, e por Almeida (2005) na mesma floresta deste estudo. Segundo estes autores, os ventos fortes e o déficit hídrico podem ter atuado conjuntamente, contribuindo para uma maior deposição de material no período de seca.

Kolm (2001), Ribeiro (2001), Rodrigues e Leitão Filho (2001), Arato et al. (2003) e Vital et al. (2004) relatam em seus trabalhos que a fração foliar é o componente principal e quantitativamente determinante de toda a serrapilheira produzida. Portanto, o padrão de produção mensal dessa fração acompanha basicamente o padrão de produção mensal da serrapilheira total. Luizão e Schubart (1986), Luizão (1989) e Souza (2004) concordam que a dinâmica da serrapilheira na floresta tropical úmida é claramente sazonal com maior queda da serrapilheira na estação seca.

\section{DINÂMICA MENSAL DA SERRAPILHEIRA ACUMULADA NO SOLO}

A variação mensal para serrapilheira acumulada oscilou de $3.527,6$ a $6.494,0 \mathrm{~kg} \cdot \mathrm{ha}^{-1}$ totalizando um valor anual de $61.453,3 \mathrm{~kg} \cdot \mathrm{ha}^{-1}$ com pico de acúmulo no período seco no mês de junho, com valor médio mensal igual a 6.494,0 $\mathrm{kg} \cdot \mathrm{ha}^{-1} \mathrm{e}$ no período de chuva, com pico de acúmulo no mês de janeiro, cujo valor médio mensal igual a 5.790,5 kg.ha-1. Os meses com menor acúmulo foram maio e dezembro, início da seca e início do período chuvoso respectivamente (Figura 4). Para a serrapilheira acumulada no solo não foi verificada variação significativa entre os períodos de seca e de chuva $(t$ $=1,55, \mathrm{P}=0,10)$.

As frações que mais se destacaram na serrapilheira acumulada foram folhas com $60 \%$, presentes em todos os meses de coleta, seguida das frações galhos $30 \%$, frutos $6 \%$ 
e flores $4 \%$, seguindo a mesma tendência observada para a serrapilheira total produzida (Figura 5).

A ligeira queda no acúmulo de serrapilheira registrada no mês de dezembro pode estar associada à alta atividade decompositora neste mês, constatada no experimento de decomposição deste trabalho. Para Valentini (2004), o acúmulo de serrapilheira no solo é regulado pela quantidade de material que cai da parte aérea das plantas e por sua taxa de decomposição, que vai enriquecendo o solo à medida que vão ocorrendo os processos de decomposição. A fração galhos, tanto para serrapilheira acumulada, como para a produzida apresentou pouca contribuição na conformação da serrapilheira total (menos de 30\%). Costa (1999) e Ribeiro (2001) em estudos feitos no Pantanal também constataram uma baixa representação da fração galhos na serrapilheira.

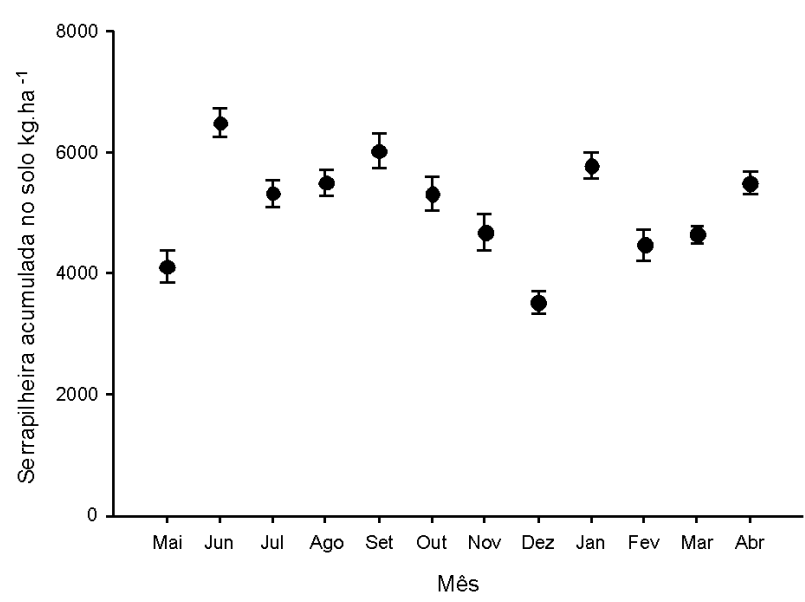

Figura 4 - Serrapilheira acumulada no solo na Floresta de Transição AmazôniaCerrado entre maio de 2004 a abril de 2005.

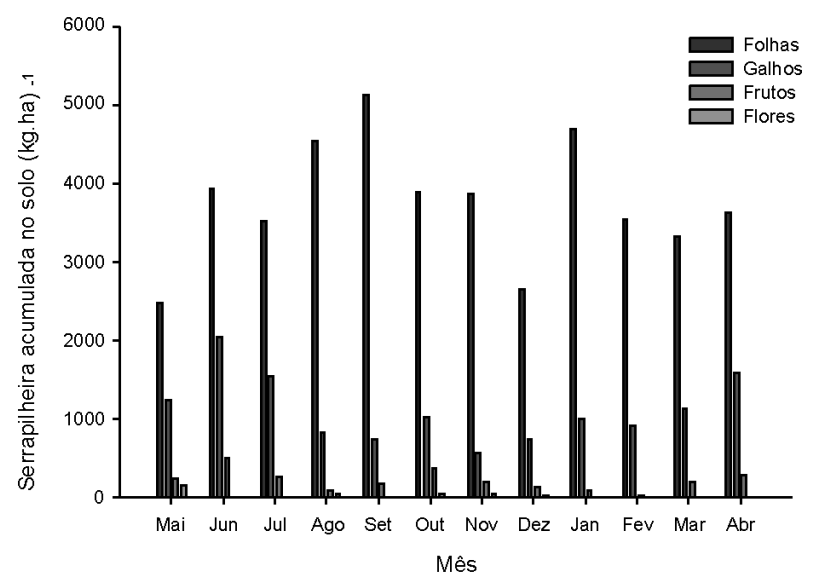

Figura 5 - Frações de serrapilheira acumulada no solo na Floresta de Transição Amazônia-Cerrado entre maio de 2004 a abril de 2005.
Quando se tomam os dados de serrapilheira produzida e a serrapilheira acumulada no solo para todos os meses do ano, ou separado por estações, não se verifica qualquer correlação significativa entre estas variáveis (para todo ano, $r=0,22 ; \mathrm{P}=0,48$; para o período seco, $\mathrm{r}=0,13 ; P=0,79 \mathrm{e}$ para o período chuvoso, $r=0,10 ; P=0,84)$. Este padrão já era de se esperar, pelo fato de que a variabilidade temporal da serrapilheira produzida (C.V. $=47,3 \%)$ é muito maior que a variabilidade temporal da serrapilheira remanescente (C.V. = 16,6\%), indicando dinâmicas distintas para ambos os processos. Nesse sentido, encontram-se valores elevados de serrapilheira produzida no período seco do ano e baixos no período chuvoso, quando para esses mesmos períodos se observam valores praticamente constantes de serrapilheira acumulada.

\section{TAXA DECOMPOSIÇÃO DA SERRAPILHEIRA EXCLUSIVA DE FOLHAS}

$\mathrm{Na}$ média, para as quatro espécies conjuntamente, o processo de decomposição das folhas foi mais intenso no período chuvoso $(35,7 \%)$ em comparação ao período de seca $(16,4 \%)$.

No período seco, a taxa de decomposição $\left(\mathrm{K}_{\mathrm{f}}\right)$ foi maior para as espécies $B$. lactescens e Tyrsodium sp, seguida das espécies $T$. schomburgkii e $P$. sagotianum (Tabela 1 ). O fato de a decomposição ser maior para as duas primeiras espécies provavelmente pode estar associado à fauna da serrapilheira $\mathrm{e}$ outros animais de maior porte uma vez que a arquitetura foliar destas espécies é bastante fina, quebrando-se com facilidade e com isto acelerando o processo de decomposição. Estes organismos, apesar de apresentarem maior atividade durante o período chuvoso, também podem agir durante o seco de menor atividade.

No período chuvoso a taxa de decomposição $\left(\mathrm{K}_{\mathrm{f}}\right)$ foi maior, em ordem decrescente de espécies, em $T$. schomburgkii, Tyrsodium sp, B. lactescens e P. sagotianum. $P$. sagotianum apresentou, tanto no período seco quanto no chuvoso, maior resistência à decomposição (Tabela 1). Este comportamento provavelmente deve estar associado a características morfofisiológicas desta espécie (tamanho, espessura, presença de lignina), as quais podem dificultar o processo de decomposição.

Segundo Luizão e Schubart (1987) e Luizão (1989), a taxa de decomposição é muito acelerada durante a estação chuvosa, quando a ação de cupins (e de outros invertebrados do solo) e o desenvolvimento de raízes finas são muito mais intensos. Para Luizão e Schubart (1986), em seu trabalho na Amazônia central, a intensa atividade de remoção da matéria orgânica por macro-artrópodos principalmente cupins do gênero Sintermes, parecem ser os fatores determinantes da diferença da perda de peso da serrapilheira na época chuvosa em relação à seca. 
Tabela 1 - Constante de decomposição da serrapilheira exclusiva de folhas estimada para quatro espécies da floresta de transição Amazônia/Cerrado para 0 período seco e chuvoso.

\begin{tabular}{lcccccc}
\hline \multirow{2}{*}{ Espécie } & \multicolumn{3}{c}{ Período Seco } & \multicolumn{3}{c}{ Período Chuvoso } \\
\cline { 2 - 7 } & $K_{f}\left(d^{-1}\right)$ & $\mathrm{R}^{2}$ & $T_{r 5 \% \%}(d)$ & $K_{f}\left(d^{-1}\right)$ & $\mathrm{R}^{2}$ & $T_{r 50 \%}(d)$ \\
\hline Tovomita schomburgkkii & $0,0013 \pm 0,0003$ & 0,5208 & 533,2 & $0,0119 \pm 0,0025$ & 0,0446 & 58,2 \\
Brosimium lactescens & $0,0050 \pm 0,0017$ & 0,1987 & 138,6 & $0,0102 \pm 0,0010$ & 0,7961 & 68,0 \\
Thyrsodium sp & $0,0032 \pm 0,0011$ & 0,0000 & 216,6 & $0,0102 \pm 0,0017$ & 0,2214 & 68,0 \\
Protium sagotianum & $0,0010 \pm 0,0002$ & 0,6227 & 693,1 & $0,0061 \pm 0,0007$ & 0,7720 & 113,6 \\
\hline
\end{tabular}

Nota: $K_{t}=$ constante de decomposição, $\mathrm{R}^{2}=$ coeficiente de determinação e $T_{r 5 \%}=$ tempo de decomposição de $50 \%$ do material. Para a variável $K_{t}$, os valores apresentados são as médias seguidas dos seus respectivos intervalos de confiança a $95 \%$ de probabilidade.

Estes animais ajudam na quebra de substâncias de difícil degradação, como a lignina e caracterizando-se por cortar e carregar discos bem arredondados das folhas da serrapilheira para o interior do solo.

VARIAÇÃO TEMPORAL DOS TEORES DE NITROGÊNIO (N), FÓSFORO (P) E CARBONO (C) NAS FOLHAS EM DECOMPOSIÇÃO DURANTE OS PERÍODOS SECO E CHUVOSO

Os teores de nitrogênio, fósforo e carbono verificados inicialmente antes do material vegetal ser levado a campo para o início dos experimentos estão identificados na Tabela 2. do tecido vegetal. Luizão e Schubart (1986) constataram que a concentração de nitrogênio nas folhas em decomposição apresenta oscilaçôes no decorrer da permanência do material no solo. Estes últimos autores registraram aumentos na concentração de nitrogênio, originado pelos resíduos do solo, juntamente com a excreção dos microorganismos da fauna do solo e da adição pela água da chuva.

Em geral, para todas as espécies, o fósforo remanescente nas folhas em decomposição apresentou oscilações, sendo o maior incremento registrado nas espécies $B$. lactescens e $P$.

Tabela 2 - Teores originais de nitrogênio, fósforo e carbono presentes nas folhas de quatro espécies da floresta de transição Amazônia/Cerrado antes do início do experimento.

\begin{tabular}{lcccccc}
\hline \multirow{2}{*}{ Espécie } & \multicolumn{3}{c}{ Teor Foliar dos Elementos Químicos segundo a época do ano } \\
\cline { 2 - 7 } & \multicolumn{2}{c}{ Nitrogênio $\left(\mathrm{mg} \mathrm{g}^{-1}\right)$} & \multicolumn{2}{c}{ Fósforo $\left(\mathrm{mg} \mathrm{g}^{-1}\right)$} & \multicolumn{3}{c}{ Carbono $\left(\mathrm{g} \mathrm{kg}^{-1}\right)$} \\
\cline { 2 - 7 } & Período de Seca & Período Chuvoso & Período de Seca & Período Chuvoso & Período de Seca & Período Chuvoso \\
\hline Tovomita schomburgkkii & 10,7 & 9,3 & 7,0 & 12,1 & 570,19 & 580,05 \\
Brosimium lactescens & 8,1 & 8,6 & 9,4 & 8,4 & 499,08 & 572,03 \\
Thyrsodium sp & 16,6 & 12,7 & 27,3 & 13,1 & 580,79 & 580,12 \\
Protium sagotianum & 6,3 & 16,0 & 9,3 & 9,0 & 574,31 & 584,26 \\
\hline
\end{tabular}

A proporção de nitrogênio remanescente nas folhas em decomposição das quatro espécies estudadas na estação chuvosa aumentou no período de 60 dias, posteriormente houve uma diminuição e novamente um aumento. Já no período da seca houve redução gradual do nitrogênio até o fim do experimento em todas as espécies. Entre as espécies, $P$. sagotianum apresentou a menor proporção remanescente de nitrogênio em todos os períodos (Figura 6).

Segundo Moraes et al. (1999) e Rodrigues e Leitão Filho (2001), a concentração de nitrogênio e fósforo originada pelo processo de decomposição da serrapilheira pode ser diferente em cada ecossistema, de acordo com propriedades do ambiente. A queda e a decomposição de serrapilheira fina representam uma maior entrada anual de fósforo para o ecossistema, enquanto as chuvas representam a maior fonte de nitrogênio (Luizão, 1989).

Aidar e Joly (2003) em seu estudo com mata ciliar no estado de São Paulo sugerem que o decaimento da concentração de nitrogênio pode estar associado aos componentes estruturais

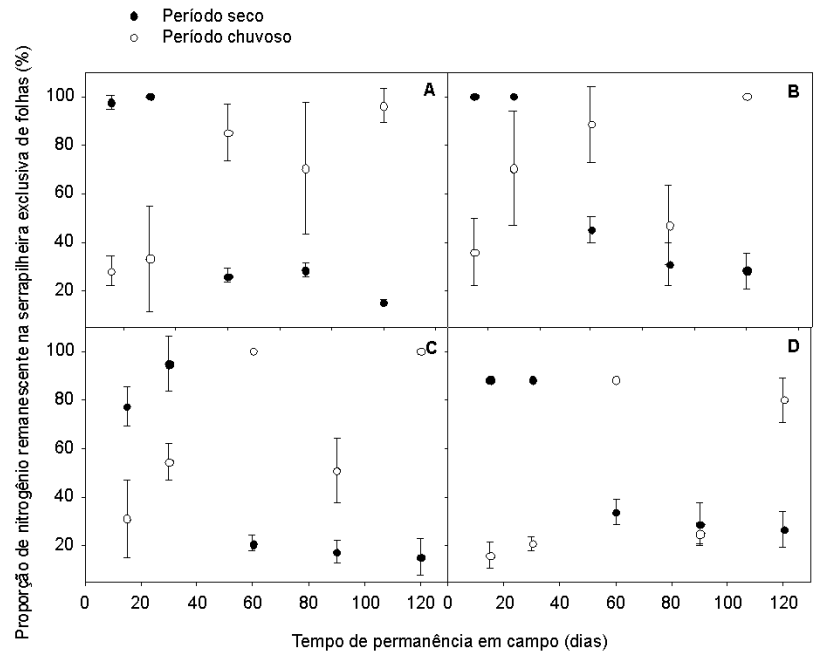

Figura 6 - Variação temporal de nitrogênio remanescente presente na serrapilheira exclusiva de folhas das espécies T. schomburgkii (A), $B$. lactescens (B), Tyrsodium sp (C) e P. sagotianum (D), no período seco de 2004 e no chuvoso de 2005, na floresta de transição Amazônia/Cerrado. 
sagotianum em torno dos 100 dias de experimento no período chuvoso. No período de seca o fósforo apresentou um pequeno aumento gradual em todas as espécies (Figura 7). Apesar dos dados iniciais de fósforo apresentarem valores que suscitem certa dúvida com relação à sua exatidão, a tendência de enriquecimento pode ser considerada como um fato passível de ocorrência e pode estar associada à ação microbiana, principalmente com as micorrizas.

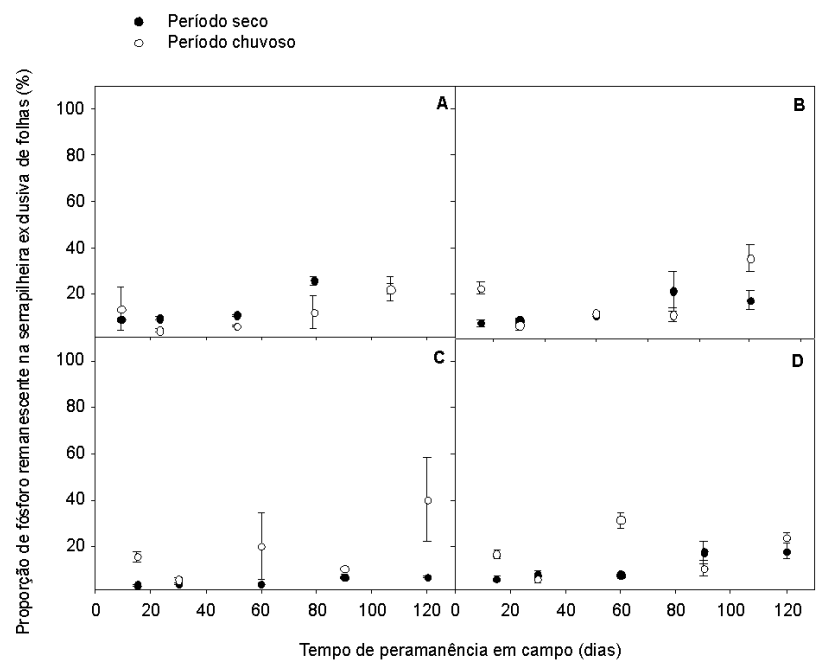

Figura 7 - Variação temporal de fósforo remanescente presente na serrapilheira exclusiva de folhas das espécies $T$. schomburgkii (A), B. lactescens (B), Tyrsodium sp (C) e P. sagotianum (D), no período seco de 2004 e chuvoso de 2005, na floresta de transição Amazônia/Cerrado.

Aidar e Joly (2003) evidenciaram em suas pesquisas que o fósforo apresentou um comportamento complexo, não caracterizando decaimento. Ribeiro (2001) no Pantanal de Mato Grosso constatou um aumento gradativo de concentração de fósforo em seu material ao longo da pesquisa.

De acordo com o método utilizado neste trabalho, a lavagem prévia do material vegetal para ser analisado foi empregada justamente para evitar riscos de contaminação, podendo-se, portanto, descartar essa hipótese para o presente caso. Considerando que não foi possível realizar qualquer análise da participação relativa de microorganismos no enriquecimento do material vegetal analisado, não se pode testar esta hipótese.

A concentração de carbono remanescente no período seco apresentou redução após 15 dias e incremento depois de 60 dias. Durante o período chuvoso a concentração de carbono nas folhas esteve em torno de $90 \%$ em todas as espécies, apresentando redução no fim do experimento apenas para as espécies T. schomburgkii e B. lactescens (Figura 8). Este elemento não apresentou diferença significativa de concentração nos dois períodos estudados. Resultados semelhantes foram evidenciados por Borém e Ramos (2002) na Mata Atlântica.

A análise da variabilidade dos nutrientes indicou, pela ausência de ajuste exponencial da regressão linear, que o tempo de retorno de um determinado nutriente deixa de existir quando há incremento na concentração do nutriente à medida que o material vegetal ficou exposto, como ocorreu com o fósforo em todas as espécies nos períodos seco e chuvoso e o nitrogênio no período chuvoso. Por meio do ajuste linear verificou-se que em alguns casos (carbono) houve significância para o modelo, ainda que com um R2 muito baixo, indicando alta variabilidade dos dados (Tabela 3).

Considerando que as espécies de plantas selecionadas neste trabalho representem a população da floresta de transição analisada, a contribuição relativa de suas folhas no retorno de nitrogênio, fósforo e carbono para o solo nesse ambiente pode ser estimado considerando a média dos valores originais destes elementos contidos no material vegetal, antes de serem expostas para decompor, e da proporção de folhas contidas na serrapilheira produzida total. Assim, observa-se que o potencial de retorno para o nitrogênio é de $70,3 \mathrm{~kg} \mathrm{ha}^{-1} \mathrm{ano}^{-1}$, para o fósforo $76,1 \mathrm{~kg} \mathrm{ha}^{-1}$ ano $^{-1}$ e para o carbono 3,5 ton ha ${ }^{-1}$ $a^{-1}$. Entretanto, cabe ressaltar que estes valores são apenas uma aproximação e que na verdade não ocorrem de fato, pois existem ainda outros fatores não considerados, tais como a perda destes elementos para a atmosfera e a contribuição relativa da chuva no processo de enriquecimento.

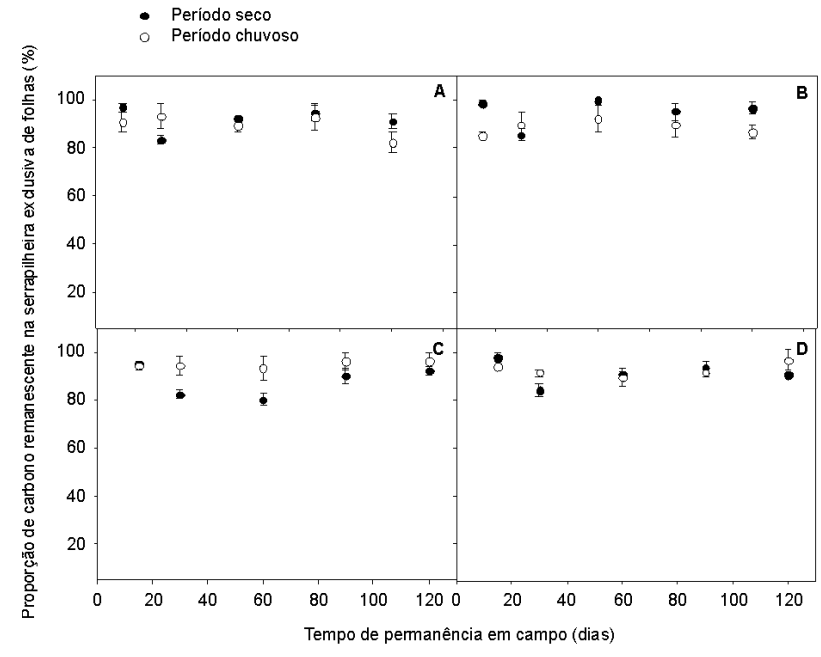

Figura 8 - Variação temporal de carbono remanescente presente na serrapilheira exclusiva de folhas das espécies T. schomburgkii (A), $B$. lactescens (B), Tyrsodium sp (C) e P. sagotianum (D), no período seco de 2004 e no chuvoso de 2005 na floresta de transição Amazônia/Cerrado. 
Tabela 3 - Tempo de retorno do carbono, fósforo e nitrogênio presente na serrapilheira exclusiva de folhas estimados para quatro espécies da floresta de transição Amazônia/Cerrado durante o período de seca de 2004 e chuvoso de 2005.

\begin{tabular}{|c|c|c|c|c|c|c|}
\hline & \multirow{2}{*}{\multicolumn{2}{|c|}{ Parâmetros Avaliados }} & \multicolumn{4}{|c|}{ Espécie } \\
\hline & & & Tovomita schomburgkkii & Brosimium lactescens & Thyrsodium sp & Protium sagotianum \\
\hline \multirow{8}{*}{ 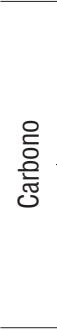 } & \multirow{4}{*}{$\begin{array}{l}\text { 응 } \\
\text { 응 } \\
\text { 은 }\end{array}$} & $\mathrm{T}_{\mathrm{r} 50 \%}$ & 566,09 & $7.949,16$ & - & - \\
\hline & & $\mathrm{R}^{2}$ & 0,21 & 0,0013 & 0,06 & 0,17 \\
\hline & & Coeficiente Linear & $93,85 \pm 3,98$ & $89,03 \pm 3,839$ & $93,47 \pm 2,85$ & $90,39 \pm 2,89$ \\
\hline & & Coeficiente Angular & $-0,08 \pm 0,07$ & $-0,0056 \pm 0,07$ & $0,03 \pm 0,05$ & $0,047 \pm 0,048$ \\
\hline & \multirow{4}{*}{ 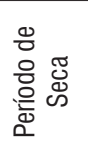 } & $\mathrm{T}_{\mathrm{r} 50 \%}$ & - & - & - & $28.600,55$ \\
\hline & & $\mathrm{R}^{2}$ & 0,02 & 0,05 & 0,02 & 0,0002 \\
\hline & & Coeficiente Linear & $90,21 \pm 4,5$ & $93,00 \pm 4,43$ & $87,04 \pm 4,85$ & $91,52 \pm 4,09$ \\
\hline & & Coeficiente Angular & $0,018 \pm 0,058$ & $0,03 \pm 0,06$ & $0,02 \pm 0,06$ & $-0,0016 \pm 0,057$ \\
\hline \multirow{8}{*}{ 음 } & \multirow{4}{*}{ 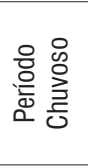 } & $\mathrm{T}_{\mathrm{r} 50 \%}$ & - & - & - & - \\
\hline & & $\mathrm{R}^{2}$ & 0,34 & 0,35 & 0,366 & 0,056 \\
\hline & & Coeficiente Linear & $4,74 \pm 5,27$ & $7,90 \pm 7,16$ & $4,80 \pm 9,66$ & $14,04 \pm 7,82$ \\
\hline & & Coeficiente Angular & $0,14 \pm 0,08$ & $0,20 \pm 0,12$ & $0,28 \pm 0,16$ & $0,07 \pm 0,13$ \\
\hline & \multirow{4}{*}{ 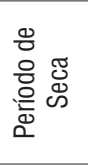 } & $\mathrm{T}_{\mathrm{r} 50 \%}$ & - & - & - & - \\
\hline & & $\mathrm{R}^{2}$ & 0,73 & 0,53 & 0,77 & 0,70 \\
\hline & & Coeficiente Linear & $5,52 \pm 3,07$ & $5,63 \pm 3,57$ & $2,45 \pm 0,67$ & $3,71 \pm 2,51$ \\
\hline & & Coeficiente Angular & $0,16 \pm 0,04$ & $0,11 \pm 0,05$ & $0,04 \pm 0,009$ & $0,12 \pm 0,034$ \\
\hline \multirow{7}{*}{$\begin{array}{l}\text { 을 } \\
\text { 융 } \\
\text { 올 } \\
\text { 旁 }\end{array}$} & \multirow{4}{*}{ 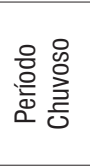 } & $\mathrm{T}_{\mathrm{r} 50 \%}$ & 0,48 & 0,20 & 0,27 & 0,25 \\
\hline & & $\mathrm{R}^{2}$ & $0,71 \pm 0,32$ & $0,40 \pm 0,36$ & $0,49 \pm 0,36$ & $0,5770 \pm 0,42$ \\
\hline & & Coeficiente Linear & $28,30 \pm 18,13$ & $46,99 \pm 20,49$ & $42,81 \pm 19,99$ & $23,56 \pm 24,74$ \\
\hline & & Coeficiente Angular & $0,71 \pm 0,32$ & $0,40 \pm 0,36$ & $0,49 \pm 0,36$ & $0,57 \pm 0,42$ \\
\hline & \multirow{3}{*}{ 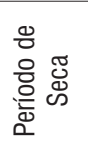 } & $\mathrm{T}_{\mathrm{r} 50 \%}$ & 54,6 & 70,7 & 44,1 & 68,6 \\
\hline & & $\mathrm{R}^{2}$ & 0,75 & 0,79 & 0,75 & 0,76 \\
\hline & & $K_{r}\left(d^{-1}\right)$ & $0,0127 \pm 0,0035$ & $0,0098 \pm 0,0023$ & $0,016 \pm 0,0038$ & $0,01 \pm 0,0024$ \\
\hline
\end{tabular}

\section{CORRELAÇÃO ENTRE A DECOMPOSIÇÃO DAS FOLHAS E 0 RETORNO DE NUTRIENTES}

Analisando a relação da taxa de decomposição com o retorno do nitrogênio (Tabela 4), verificou-se que, para este nutriente, há indícios de um processo de lixiviação durante o período seco do ano (correlaçẫo positiva), ao passo que durante o período chuvoso há um enriquecimento do material vegetal remanescente (correlação negativa). Este aspecto se apresenta estatisticamente significativo para todas as espécies no período de seca, e apenas a espécie P. sagotianum no período chuvoso. Devido sua mobilidade, provavelmente a umidade do solo durante o período seco seja suficiente para possibilitar a perda desse elemento para o solo, ao passo que durante o período chuvoso, pode haver incorporação do mesmo devido à própria água da chuva (embora não tenha sido feita neste estudo uma análise da qualidade da água da chuva, impossibilitando aferir sobre esta hipótese) e da lavagem da copa das plantas.

Nota-se que para o fósforo, ocorre um enriquecimento do material vegetal remanescente à medida que avança o processo de decomposição da matéria orgânica. Este aspecto se apresenta estatisticamente significativo para todas as espécies no período de seca, porém não é significativo para nenhuma das espécies estudadas no período chuvoso (Tabela 4).

Para o carbono, somente para a espécie $P$. sagotianum e no período chuvoso verificou-se uma correlação significativa entre as variáveis analisadas (Tabela 4). Neste caso, seria interessante avaliar a natureza bioquímica dos materiais estruturais constitutivos das folhas a fim de se ter algum indício acerca de que isto é devido à existência de alguma facilidade com que estes podem ser lixiviados do material vegetal em decomposição.

\section{CONCLUSÕES}

A produção de serrapilheira apresentou variaçóes sazonais com maior produção no período de seca e predomínio da fração foliar em ambos os períodos. A serrapilheira acumulada não apresentou diferenças significativas entre o período de seca e chuva e não apresentou correlação significativa com a produção de serrapilheira.

A serrapilheira exclusiva de folhas apresentou uma maior decomposição no período chuvoso. O potencial de retorno 
Tabela 4 - Correlação entre a fração de matéria seca remanescente da serrapilheira exclusiva de folhas e a proporção dos teores de nitrogênio, fósforo e carbono atuais (N, P, C) em relação aos iniciais (NO, P0, C0) durante os períodos seco de 2004 e chuvoso de 2005 na floresta de transição Amazônica/Cerrado.

\begin{tabular}{|c|c|c|c|c|c|c|c|}
\hline \multicolumn{2}{|c|}{ Variáveis } & \multicolumn{3}{|c|}{$\begin{array}{l}\text { Material Vegetal Remanescente no Período } \\
\qquad \text { Seco }\end{array}$} & \multicolumn{3}{|c|}{$\begin{array}{c}\text { Material Vegetal Remanescente no Período } \\
\text { Chuvoso }\end{array}$} \\
\hline Espécie & Proporção do Nutriente & $r$ & $\mathrm{P}$ & $\mathrm{n}$ & $r$ & $P$ & $\mathrm{n}$ \\
\hline \multirow{3}{*}{ T. schomburgkkii } & $\mathrm{N} / \mathrm{N}_{0}$ & 0,5515 & 0,0096 & 21 & $-0,4781$ & 0,0181 & 24 \\
\hline & $\mathrm{P} / \mathrm{P}_{0}$ & $-0,6992$ & 0,0002 & 23 & $-0,4485$ & 0,0318 & 23 \\
\hline & $\mathrm{C} / \mathrm{C}_{0}$ & $-0,1293$ & 0,5662 & 22 & 0,1782 & 0,4047 & 24 \\
\hline \multirow{3}{*}{ B. lactescens } & $\mathrm{N} / \mathrm{N}_{0}$ & 0,8383 & 0,0000 & 21 & $-0,3293$ & 0,1450 & 21 \\
\hline & $\mathrm{P} / \mathrm{P}_{0}$ & $-0,7825$ & 0,0000 & 21 & $-0,4450$ & 0,0380 & 22 \\
\hline & $\mathrm{C} / \mathrm{C}_{0}$ & $-0,1247$ & 0,5804 & 22 & $-0,0376$ & 0,8682 & 22 \\
\hline \multirow{3}{*}{ Thyrsodium sp } & $\mathrm{N} / \mathrm{N}_{0}$ & 0,8738 & 0,0000 & 24 & $-0,4931$ & 0,0168 & 23 \\
\hline & $\mathrm{P} / \mathrm{P}_{0}$ & $-0,8065$ & 0,0000 & 22 & $-0,4739$ & 0,0223 & 23 \\
\hline & $\mathrm{C} / \mathrm{C}_{0}$ & 0,0016 & 0,9942 & 23 & $-0,2503$ & 0,2613 & 22 \\
\hline \multirow{3}{*}{ P. sagotianum } & $\mathrm{N} / \mathrm{N}_{0}$ & 0,6517 & 0,0010 & 22 & $-0,5273$ & 0,0097 & 23 \\
\hline & $\mathrm{P} / \mathrm{P}_{0}$ & $-0,7781$ & 0,0000 & 22 & $-0,2947$ & 0,1831 & 22 \\
\hline & $\mathrm{C} / \mathrm{C}_{0}$ & 0,0421 & 0,8525 & 22 & $-0,5052$ & 0,0231 & 20 \\
\hline
\end{tabular}

Nota: $r=$ coeficiente de correlação linear, $P=$ nivel de probabilidade, $n=$ número de dados analisados.

para o nitrogênio foi da ordem de 70,3 $\mathrm{kg} \mathrm{ha}^{-1}$, para o fósforo $76,1 \mathrm{~kg} \mathrm{ha}^{-1}$ e para o carbono 3,5 ton ha- ${ }^{-1}$ ano $^{-1}$.

A correlação entre a decomposição exclusiva de folhas e o retorno de nitrogênio e fósforo foi significativa para todas as espécies no período de seca, já para o carbono a correlação foi significativa apenas para a espécie Protium sagotianum no período chuvoso.

\section{AGRADECIMENTOS}

A CAPES pela bolsa concedida ao primeiro autor, ao Projeto LBA (Experimento de Grande Escala da BiosferaAtmosfera na Amazônia) por fomentar a pesquisa na área de Transição Amazônia-Cerrado e a Universidade Federal de Mato Grosso pela disponibilização dos laboratórios.

\section{BIBLIOGRAFIA CITADA}

Aidar, M.P.M.; Joli, C.A. 2003. Dinâmica da produção e decomposição de serrapilheira do araribá (Centrolobium tomentosum Guill. Ex Benth. - Fabaceae) em uma mata ciliar, Rio Jacaré-Pepira, São Paulo. Revista. Brasileira de Botânica, 26 (2): 193-202.

Allen, S.E. 1989. Chemical analysis of ecological materials. 2 Ed. Blackwell Scientific Publications, London. 368pp.

Almeida, E.D. 2005. Retorno de nitrogênio e fósforo em floresta de transição no noroeste de Mato Grosso. Dissertação de Mestrado, Universidade Federal de Mato Grosso, Cuiabá. 75pp.

Arato, H.D.; Martins, S.V.; Ferrari, S.H.S. 2003. Produção e decomposição de serrapilheira em um sistema agroflorestal implantado para recuperação de área degradada em Viçosa - MG. Revista Árvore, 27 (5): 715-721.

Boinskis, S. 1989. Seasonal patterns in a tropical lowland forest. Biotropica, 21(33): 223-233.
Bocock, K.L.; Gilbert, O.J.W. 1957. The disappearance of litter under different woodland conditions. Plant and Soil, 9(1): 179-185.

Borém, R.A.T.; Ramos, D.P. 2002. Variação estacional e topográfica de nutrientes na serrapilheira de um fragmento de Mata Atlântica. Revista Cerne, 8 (2): 42-59.

Costa, S.C. 1999. Aspectos da produção e decomposição de serrapilheira em um trecho de floresta ripária do Pantanal de Barão de MelgaçoMT. Monografia de Conclusão de Curso, Universidade Federal de Mato Grosso, Cuiabá. 40pp.

Dantas, M.; Phillipson, J. 1989. Litterfall and litter nutrient content in primary and secundary Amazonian "Terra firme" rain forest. Journal of Tropical Ecology, 5: 27-36.

De Maman, A.P. 2006. Produção e decomposição de serrapilheira em duas áreas do bioma Cerrado (Cerradão e Mata de Galeria) no sudoeste de Mato Grosso. Monografia de Conclusão de Curso, Universidade do Estado de Mato Grosso, Tangará da Serra. 73pp.

Klinge, H.; Rodrigues, W.A. 1968. Litter production in an area of Amazonian terra firme forest. Part.I: Litter-fall, organic carbon and total nitrogen contents of litter. Amazoniana, 1 (4): 287 302.

Kolm, L. 2001. Ciclagem de nutrientes e variaçôes do microclima em plantaçôes de Eucalyptus grandis Hill ex maiden manejadas através de desbastes progressivos. Dissertação de mestrado, Escola Superior de Agricultura "Luiz de Queiroz". Universidade de São Paulo, São Paulo. 73pp.

Leitão Filho, H.F.; Pagano, S.N.; Cesar, O.; Timoni, J.L.; Rueda, J.J. 1993. Ecologia da mata atlântica em Cubatão, SP. EDUNESP/ EDUNICAMP, São Paulo. 86 pp.

Luizão, F.J.; Schubart, H.O.R. 1986. Produção e decomposição de liteira em floresta de terra firme da Amazônia Central. Acta Limnol. Brasil, 1: 575:600. 
Luizão, F.J. 1989. Litter production and mineral element input to the forest floor in a central Amazonian forest. Geol. Journal, 19:407- 417.

Luizão, F.J.; Schubart, O.R.H. 1987. Litter production and decomposition in a terra-firme forest of Central Amazonia. Experientia, 43:259-265.

Martins, S.V.; Rodrigues, R.R. 1999. Produção de Serrapilheira em clareiras de uma floresta estacional semidecidual no Município de Campinas, SP. Revista Brasileira de Botânica, 22 (3): 405-412.

Moraes, R.M.; Deletti, W.B.C.; Struffaldi-de Vuono, Y. 1999. Litterfall and litter nutrient content in two Brazilian Tropical Forests. Revista Brasileira de Botânica, 22 (1): 9-16.

Morellato, P.C.; Leitão Filho, H.F. 1995. Ecologia e preservação de uma floresta tropical urbana: reserva de Santa Genebra. UNICAMP, Campinas, São Paulo. 79pp.

Naeem, S.; Thompson, L.J.; Lawler, S.P.; Lawton, J.H.; Woodfin, R.M. 1995. Empirical evidence that declining species diversity may alter the performance of terrestrial ecosystems. Philosophical Transactions of the Royal Society of London, 347: 249-262.

Olson, J.S. 1963. Energy storage and the balance of producers and decomposers in ecological systems. Ecology, 44 (2): 322-331.

Pinto Jr, O.B. 2007. Efluxo de CO do solo em floresta de transição Amazônia Cerrado e em área de pastagem. Dissertação de mestrado, Universidade Federal de Mato Grosso, Cuiabá. 65pp.

Ribeiro, L. 2001. O papel da serrapilheira na dinâmica de nutrientes do Landi da Moranga, Pantanal Mato-Grossense, Brasil. Dissertação de mestrado, Universidade Federal de Mato Grosso, Cuiabá. 58pp.

Rodrigues, W.A.; Klinge, H.; Fittkau, E.J. 2000. Estrutura e funcionamento de um ecossistema florestal amazônico de terra firme junto à Reserva Florestal Walter Egler, município de Rio Preto da Eva, Amazonas, Brasil. Acta Biol. Par., 29 (1, 2, 3, 4): 219-243.
Rodrigues, R.R.; Leitão Filho, H.F. 2001. Matas Ciliares: Conservação e Recuperação. 2. ed. Fapesp, São Paulo. 320pp.

Sanches, L.; Suli, G.S.; Prinate-Filho, N.; Vourlitis, G.; Nogueira, J.S. 2005. Índice de área foliar em floresta de transição AmazôniaCerrado. Rev. Ciência Nat., 1: 37-40.

Sanches, L.; Valentini, C.M.A.; Pinto Junior, O.B.; Nogueira, J.S.; Vourlitis, G.; Biudes, M.S.; Silva, C.J.; Bambi, P.; Lobo, F.A. 2008. Seasonal and interannual litter dynamics of a tropical semideciduous forest of the southern Amazon Basin, Brazil. Journal of Geophysical Research, 113:G04007: 1-9.

Silva, C.J.; Sanches, L.; Bleich, M.E.; Lobo, F.A.; Nogueira, J.S. 2007. Produção de serrapilheira no Cerrado e Floresta de transição Amazônia-Cerrado do centro-oeste brasileiro. Acta Amazônica, 37(4): 543-548.

Souza, J.S. 2004. Dinâmica espacial e temporal do fluxo de $\mathrm{CO}_{2}$ do solo em floresta de terra firme na Amazônia central. Dissertação de Mestrado, Instituto de Pesquisas da Amazônia, Manaus. 62pp.

Tilman, D.; Wedin, D.; Knops, J. 1996. Productivity and sustainability influenced by biodiversity in grassland ecosystems. Nature, 379: 718-720.

Valentini, C.M.A. 2004. Efluxo de $\mathrm{CO}_{2}$ do solo de uma área de floresta de transição no noroeste de Mato Grosso. Dissertação de Mestrado, Universidade Federal de Mato Grosso, Cuiabá. 81pp.

Vital, A.R.T.; Guerrini, I.A.; Franken, W.K.; Fonseca, R.C.B. 2004. Produção de serrapilheira e ciclagem de nutrientes de uma floresta estacional semidecidual em zona ripária. Revista Árvore, 28 (6): 793-800.

Vourlitis, G.L.; Priante Filho, N.; Hayashi, M.M.S.; Nogueira, J.S.; Caseiro, F.T.; Campelo Jr, J. H. 2001. Seasonal variations en the net ecosystem CO2 exchange of a mature Amazonian transitional tropical Forest (Cerradão). Functional ecology, 15: 388-395.

Recebido em 21/11/2008

Aceito em 27/05/2009 